\title{
STRONG CONVERGENCE OF DISCRETE DG SOLUTIONS OF THE HEAT EQUATION
}

\author{
VIVETTE GIRAULT * , JIZHOU LI , AND BEATRICE RIVIÈRE ${ }^{\dagger}$
}

\begin{abstract}
A convergence analysis to the weak solution is derived for interior penalty discontinuous Galerkin methods applied to the heat equation in two and three dimensions under general mixed boundary conditions. Strong convergence is established in the DG norm, as well as in the $L^{p}$ norm, in space and in the $L^{2}$ norm in time.
\end{abstract}

Key words. weak solution, mixed boundary conditions, backward Euler, $L^{p}$ bound, $L^{p}$ convergence

1. Introduction. This work is dedicated to the strong convergence of the discrete discontinuous Galerkin (DG) solutions to the weak solution of the heat equation. Analysis is given for all three variants of the interior penalty DG methods, and for general mixed boundary conditions. In addition, $L^{p}$ bounds and convergence for discrete broken Sobolev spaces are obtained.

In [4], strong convergence is shown for the steady Poisson problem with homogeneous Dirichlet boundary condition; it is obtained by introducing an associated discrete gradient operator and working directly on the scheme and PDE. Here in order to address the heat equation, we first derive a bound for the time-derivative in the $L^{2}$ norm in time and in a dual norm in space. We then work also directly on the scheme and PDE. This gives strong convergence of the solutions in the DG and $L^{2}$ norms, in space and in the $L^{2}$ norm in time. In addition, convergence in the $L^{p}$ norm in space is recovered by lifting the DG functions with a non-conforming interpolant and a conforming regularization. This approach is in the spirit of that used by Brenner in [1]; it is robust, valid in 2D or 3D; and it applies to general mixed boundary conditions. Since the model problem is linear, the analysis only uses linear techniques, and in particular, does not need an Aubin-Lions lemma. However, apart from this compactness lemma, the tools used in this work can give an insight on what could be done for the analysis of DG methods applied to some nonlinear parabolic problems.

The outline of the paper is as follows. The model problem is described in the next section. The discrete scheme for the PDE is introduced and the convergence result of the broken gradient is stated in Section 3. Intermediate results are obtained in Sections 4 and 5. General $L^{p}$ convergence results in broken Sobolev spaces are derived in Section 6. The proof of the convergence result for the broken gradient is given in Section 7. Some technical proofs are outlined in an appendix.

\footnotetext{
${ }^{*}$ Paris VI, visiting professor at Rice

${ }^{\dagger}$ Department of Computational and Applied Mathematics, Rice University, Houston, TX 77005. Supported in part by NSF-DMS 1318348
} 
2. Model problem and weak formulation. Let $\Omega$ be a bounded domain of $\mathbb{R}^{d}$, $d=2,3$, with a Lipschitz boundary $\partial \Omega$ partitioned into two disjoint parts $\partial \Omega=\Gamma_{D} \cup \Gamma_{N}$. The regions $\Gamma_{D}$ and $\Gamma_{N}$ need not be connected, but we assume that the number of connected components of $\Gamma_{D}$ and $\Gamma_{N}$ is finite. Let $Q_{T}=\Omega \times(0, T)$ denote the space-time cylinder. The exact problem reads: Find $u$ such that

$$
\begin{gathered}
\frac{\partial u}{\partial t}-\Delta u=f, \quad \text { in } Q_{T}, \\
u=0, \quad \text { on } \Gamma_{D} \times(0, T), \\
\nabla u \cdot \mathbf{n}_{\Omega}=0, \quad \text { on } \Gamma_{N} \times(0, T), \\
u=u_{0}, \quad \text { in } \Omega \text { at time } t=0,
\end{gathered}
$$

where $\mathbf{n}_{\Omega}$ is the unit outward normal vector to $\partial \Omega$. The analysis below includes the cases where $\Gamma_{N}=\partial \Omega$ or $\Gamma_{D}=\partial \Omega$.

Define the space

$$
X=H_{0, \Gamma_{D}}^{1}(\Omega)=\left\{v \in H^{1}(\Omega), v=0 \text { on } \Gamma_{D}\right\},
$$

equipped with the norm of $H^{1}(\Omega)$, and let $X^{\prime}$ denote its dual space. The $L^{2}$ inner-product over a generic domain $\mathcal{O}$ is denoted by $(\cdot, \cdot)_{\mathcal{O}}$, or simply by $(\cdot, \cdot)$ when there is no ambiguity, and the duality pairing between the dual $X^{\prime}$ and $X$ by $\langle\cdot, \cdot\rangle$.

Assume that $f$ belongs to $L^{2}\left(Q_{T}\right)$ and that $u_{0}$ belongs to $L^{2}(\Omega)$. The weak formulation is: find $u \in L^{2}(0, T ; X) \cap L^{\infty}\left(0, T ; L^{2}(\Omega)\right)$ such that

$$
\int_{0}^{T}\left(\left\langle\frac{\partial u}{\partial t}, v\right\rangle+(\nabla u, \nabla v)_{\Omega}\right) d t=\int_{0}^{T}(f, v)_{\Omega} d t, \quad \forall v \in L^{2}(0, T ; X)
$$

and

$$
u(0)=u_{0}
$$

By a standard argument, see e.g. [12], it can be shown that (2.1) has exactly one solution $u$ in $L^{2}(0, T ; X) \cap L^{\infty}\left(0, T ; L^{2}(\Omega)\right)$ satisfying

$$
\frac{\partial u}{\partial t} \in L^{2}\left(0, T ; X^{\prime}\right)
$$

As is usual, we associate with (2.1) the space

$$
W(0, T)=\left\{v \in L^{2}(0, T ; X) ; \frac{\partial v}{\partial t} \in L^{2}\left(0, T ; X^{\prime}\right)\right\},
$$

equipped with the graph norm. It is well-known (see e.g. $[5])$ that $\mathcal{C}^{\infty}([0, T] ; X)$ is dense in $W(0, T)$ and, as $X \hookrightarrow L^{2}(\Omega) \hookrightarrow X^{\prime}$, with continuous and dense imbeddings,

$$
W(0, T) \hookrightarrow \mathcal{C}^{0}\left([0, T] ; L^{2}(\Omega)\right) .
$$

Therefore, the above initial condition is well-defined. 
3. Numerical scheme. We combine the backward Euler method in time with the interior penalty discontinuous Galerkin method in space. Let $\mathcal{E}_{h}$ be a regular (in the sense of Ciarlet [2]) partition of the domain $\bar{\Omega}$ into simplices, or quadrilaterals or hexahedra according to the dimension, constructed so that any given boundary face belongs to either $\Gamma_{D}$ or $\Gamma_{N}$. Denote by $\Gamma_{h}$ the set of interior faces. Let $h$ denote the maximum diameter of the mesh elements. For a given face $e$, let $h_{e}$ denote the diameter of the face. Let $X_{h}$ denote the finite-dimensional space of discontinuous piecewise polynomials:

$$
X_{h}=\left\{v \in L^{2}(\Omega) ;\left.v\right|_{E} \in \mathcal{P}_{r}(E), \forall E \in \mathcal{E}_{h}\right\}, \quad r \geq 1,
$$

where $\mathcal{P}_{r}(E)$ is, for simplices, $\mathbb{P}_{r}(E)$, the space of polynomials of degree less than or equal to $r$. For quadrilateral or hexaedral elements, $\mathcal{P}_{r}(E)$ is the image by the inverse transformation, i.e., $\hat{\mathbf{x}}=\mathcal{F}^{-1}(\mathbf{x})$, of polynomials of degree less than or equal to $r$ in each variable, i.e. $\mathbb{Q}_{r}(\hat{E})$. Let $N>0$ be an integer, define $\Delta t=T / N$ and $t_{n}=n \Delta t$. The fully discrete scheme is to find $u_{h}^{n+1} \in X_{h}$ satisfying

$$
\frac{1}{\Delta t}\left(u_{h}^{n+1}-u_{h}^{n}, v_{h}\right)+a_{\mathrm{DG}}\left(u_{h}^{n+1}, v_{h}\right)=\left(f^{n+1}, v_{h}\right), \quad \forall v_{h} \in X_{h}, \quad 0 \leq n \leq N-1,
$$

where

$$
f^{n+1}(x)=\frac{1}{\Delta t} \int_{t_{n}}^{t_{n+1}} f(x, t) d t, \quad \text { a.e. in } \Omega,
$$

and $u_{h}^{0}=P_{h} u_{0}$, where $P_{h}$ is the $L^{2}$ projection operator in space defined for any $v$ in $L^{2}(\Omega)$, by $P_{h} v \in X_{h}$ satisfying

$$
\left(P_{h} v, w_{h}\right)=\left(v, w_{h}\right), \quad \forall w_{h} \in X_{h} .
$$

We recall the usual notation for the DG set-up. We define the broken gradient $\nabla_{h} w_{h}$ as the piecewise gradient on each mesh element. For a given interior face $e$, a unit normal vector $\mathbf{n}_{e}$ is fixed, and its orientation is used to uniquely denote the elements that share $e$ by $E_{1}^{e}, E_{2}^{e}$. The jump and average of a function $v \in X_{h}$ across a face $e$ are given as

$$
[v]=\left.v\right|_{E_{1}^{e}}-\left.v\right|_{E_{2}^{e}}, \quad\{v\}=\left.\frac{1}{2} v\right|_{E_{1}^{e}}+\left.\frac{1}{2} v\right|_{E_{2}^{e}} .
$$

For a boundary face, the unit normal vector $\mathbf{n}_{e}$ is the outward normal $\mathbf{n}_{\Omega}$ to $\partial \Omega$, and the jump and average simply coincide with the trace. The DG bilinear form is for all $w, v$ in $X_{h}$ :

$$
\begin{aligned}
a_{\mathrm{DG}}(w, v)=\sum_{E \in \mathcal{E}_{h}}\left(\nabla_{h} w, \nabla_{h} v\right)_{E}- & \sum_{e \in \Gamma_{h} \cup \Gamma_{D}}\left(\{\nabla w\} \cdot \mathbf{n}_{e},[v]\right)_{e} \\
& +\epsilon \sum_{e \in \Gamma_{h} \cup \Gamma_{D}}\left(\{\nabla v\} \cdot \mathbf{n}_{e},[w]\right)_{e}+\sum_{e \in \Gamma_{h} \cup \Gamma_{D}} \frac{\sigma_{e}}{h_{e}}([w],[v])_{e} .
\end{aligned}
$$

The symmetrization parameter $\epsilon$ may take the values $-1,0$ or +1 . The penalty parameter $\sigma_{e} \geq 1$ may vary from face to face, it is chosen in particular so that the form $a_{\mathrm{DG}}$ is coercive 
in the case when $\epsilon=-1$ or $\epsilon=0$, see [9]. Define the semi-norms

$$
\|w\|_{X_{h}}=\left(\sum_{E \in \mathcal{E}_{h}}\|\nabla w\|_{L^{2}(E)}^{2}+\sum_{e \in \Gamma_{h} \cup \Gamma_{D}} \frac{\sigma_{e}}{h_{e}}\|[w]\|_{L^{2}(e)}^{2}\right)^{1 / 2}, \quad\|w\|_{L^{2}\left(0, T ; X_{h}\right)}=\left(\int_{0}^{T}\|w\|_{X_{h}}^{2}\right)^{1 / 2} .
$$

They are norms if $\left|\Gamma_{D}\right|>0$. For convenience, we will use the compact notation $(\cdot, \cdot)_{\Gamma_{h} \cup \Gamma_{D}}$ for the sum of $L^{2}$ inner-products over the interior and Dirichlet faces.

Standard energy-type estimates yield

$$
\sup _{1 \leq n \leq N}\left\|u_{h}^{n}\right\|_{L^{2}(\Omega)}^{2}+\Delta t \sum_{n=0}^{N-1}\left\|u_{h}^{n+1}\right\|_{X_{h}}^{2}+\sum_{n=0}^{N-1}\left\|u_{h}^{n+1}-u_{h}^{n}\right\|_{L^{2}(\Omega)}^{2} \leq C .
$$

Throughout the paper, the constant $C$ denotes a generic constant that is independent of $h$ and $\Delta t$.

3.1. Main results. The main convergence result of this paper is the strong convergence of the broken gradient $\nabla_{h} u_{h}^{n}$ to $\nabla u$ in $L^{2}$. More precisely, we prove the following theorem:

THEOREM 3.1. We have

$$
\lim _{h, \Delta t \rightarrow 0} \sum_{n=0}^{N-1} \int_{t_{n}}^{t_{n+1}}\left\|u_{h}^{n+1}-u\right\|_{X_{h}}^{2} d t=0
$$

Moreover,

$$
\lim _{h, \Delta t \rightarrow 0} \sup _{0 \leq n \leq N}\left\|u_{h}^{n}-u\left(t_{n}\right)\right\|_{L^{2}(\Omega)}=0
$$

Of course, (3.5) implies

$$
\lim _{h, \Delta t \rightarrow 0} \sum_{n=0}^{N-1} \int_{t_{n}}^{t_{n+1}}\left\|u_{h}^{n+1}-u\right\|_{L^{2}(\Omega)}^{2} d t=0 .
$$

This theorem has the following corollary:

Corollary 3.2. Let $p_{0}$ be the exponent of the Sobolev embedding of $H^{1}(\Omega)$ into $L^{p}(\Omega)$ defined by

$$
\frac{1}{p_{0}}=\frac{1}{2}-\frac{1}{d}
$$

Then, for all $p<p_{0}$

$$
\lim _{h, \Delta t \rightarrow 0} \sum_{n=0}^{N-1} \int_{t_{n}}^{t_{n+1}}\left\|u_{h}^{n+1}-u\right\|_{L^{p}(\Omega)}^{2} d t=0 .
$$

This corollary is an immediate consequence of Theorem 3.1 and the following theorem on $L^{p}$ bounds for broken Sobolev spaces: 
TheOrem 3.3. For each $p \leq p_{0}$ defined by (3.7), there is a constant $C$ independent of $h$ such that,

$$
\left\|v_{h}\right\|_{L^{p}(\Omega)} \leq C\left(\left\|v_{h}\right\|_{L^{2}(\Omega)}^{2}+\left\|v_{h}\right\|_{X_{h}}^{2}\right)^{1 / 2}, \quad \forall v_{h} \in X_{h} .
$$

In addition, if $\left|\Gamma_{D}\right|>0$, then

$$
\left\|v_{h}\right\|_{L^{p}(\Omega)} \leq C\left\|v_{h}\right\|_{X_{h}}, \quad \forall v_{h} \in X_{h},
$$

and if $\left|\Gamma_{D}\right|=0$, then

$$
\left\|v_{h}-\frac{1}{|\Omega|} \int_{\Omega} v_{h}\right\|_{L^{p}(\Omega)} \leq C\left\|v_{h}\right\|_{X_{h}}, \quad \forall v_{h} \in X_{h} .
$$

The bound (3.10) has been obtained for $p=2$ in [1], for any $p$ for $d=2$ in [7], and for any $p$ for $d=2,3$ in the case $\Gamma_{D}=\partial \Omega$ in [4]. The proof of Theorem 3.3 is in Appendix 9. Section 6 contains an additional important $L^{p}$ convergence result for broken Sobolev spaces. The proof of Theorem 3.1 is given in Section 7. It requires intermediate stability and convergence results for two functions $u_{h, \Delta t}$ and $\bar{u}_{h, \Delta t}$ introduced in the next section.

4. A priori bounds of $u_{h, \Delta t}$ and $\bar{u}_{h, \Delta t}$. It is usual to construct from the sequence $\left(u_{h}^{n}\right)_{n}$ a function $u_{h, \Delta t}$ that is continuous in time in $[0, T]$ and a function $\bar{u}_{h, \Delta t}$ that is piecewise constant in time in $] 0, T$. More precisely we define for any $n$, with $0 \leq n \leq N-1$ :

$$
\left.\left.u_{h, \Delta t}(t, x)=u_{h}^{n+1}(x) \frac{t-t_{n}}{\Delta t}-u_{h}^{n}(x) \frac{t-t_{n+1}}{\Delta t}, \quad \forall(t, x) \in \Omega \times\right] t_{n}, t_{n+1}\right]
$$

and

$$
\bar{u}_{h, \Delta t}(t, x)=u_{h}^{n+1}(x), \quad \forall(t, x) \in \Omega \times\left[t_{n}, t_{n+1}[.\right.
$$

Clearly, (3.4) is equivalent to

$$
\lim _{h, \Delta t \rightarrow 0}\left\|\bar{u}_{h, \Delta t}-u\right\|_{L^{2}\left(0, T ; X_{h}\right)}=0 .
$$

In the next section, we state and prove properties of these two functions $u_{h, \Delta t}$ and $\bar{u}_{h, \Delta t}$. The proof of (3.4) and (3.5) is given in Section 7.

We easily see that the scheme (3.1) can be rewritten as:

$$
\left.\left.\left(\partial_{t} u_{h, \Delta t}, v_{h}\right)+a_{\mathrm{DG}}\left(\bar{u}_{h, \Delta t}, v_{h}\right)=\left(\bar{f}_{\Delta t}, v_{h}\right), \quad \forall t \in\right] 0, T\right], \quad \forall v_{h} \in X_{h},
$$

where $\bar{f}_{\Delta t}$ is the piecewise constant function, equal to $f^{n+1}$ on the interval $\left.] t_{n}, t_{n+1}\right]$ for any $0 \leq n \leq N-1$.

4.1. A priori bounds of $\bar{u}_{h, \Delta t}$. The a priori bounds for the sequence $\left(u_{h}^{n}\right)_{n}$ yield several bounds for the function $\bar{u}_{h, \Delta t}$. From (3.3), there is a constant $C$ independent of $h$ and $\Delta t$ such that

$$
\left\|\bar{u}_{h, \Delta t}\right\|_{L^{\infty}\left(0, T ; L^{2}(\Omega)\right)}+\left\|\bar{u}_{h, \Delta t}\right\|_{L^{2}\left(0, T ; X_{h}\right)} \leq C .
$$

This immediately implies that

$$
\left\|\bar{u}_{h, \Delta t}\right\|_{L^{2}\left(Q_{T}\right)} \leq C .
$$


4.2. A priori bounds of $u_{h, \Delta t}$. From (3.3), the fact $u_{h, \Delta t}$ is a piecewise linear function in time, and the definition of $u_{h}^{0}$, there is a constant $C$ independent of $h$ and $\Delta t$ such that

$$
\left\|u_{h, \Delta t}\right\|_{L^{\infty}\left(0, T ; L^{2}(\Omega)\right)} \leq C
$$

This bound immediately implies

$$
\left\|u_{h, \Delta t}\right\|_{L^{2}\left(Q_{T}\right)} \leq C
$$

Next, we will derive a bound on the time derivative of $u_{h, \Delta t}$. Considering the definition of $X_{h}, P_{h}$ is a local $L^{2}$ projection in each element. It is easy to check that

$$
\left\|P_{h} v\right\|_{L^{2}\left(Q_{T}\right)} \leq\|v\|_{L^{2}\left(Q_{T}\right)}
$$

and

$$
\left\|P_{h} v\right\|_{L^{2}\left(0, T ; X_{h}\right)} \leq C\|v\|_{L^{2}\left(0, T ; H^{1}(\Omega)\right)}, \quad \forall v \in L^{2}(0, T ; X) .
$$

LEMMA 4.1. There is a constant $C$ independent of $h$ and $\Delta t$ such that

$$
\sup _{v \in L^{2}(0, T ; X)} \frac{\int_{0}^{T}\left(\partial_{t} u_{h, \Delta t}, P_{h} v\right) d t}{\|v\|_{L^{2}\left(0, T ; H^{1}(\Omega)\right)}} \leq C .
$$

Proof. Let $v$ be a function in $L^{2}(0, T ; X)$. Since $P_{h} v$ belongs to $X_{h}$, we obtain from (4.2)

$$
\int_{0}^{T}\left(\partial_{t} u_{h, \Delta t}, P_{h} v\right) d t=\int_{0}^{T}\left(\left(\bar{f}_{\Delta t}, P_{h} v\right)-a_{\mathrm{DG}}\left(\bar{u}_{h, \Delta t}, P_{h} v\right)\right) d t
$$

The first term in the second line is simply bounded as

$$
\sum_{n=0}^{N-1} \int_{t_{n}}^{t_{n+1}}\left(f^{n+1}, P_{h} v\right) d t \leq\|f\|_{L^{2}\left(Q_{T}\right)}\|v\|_{L^{2}\left(Q_{T}\right)} \leq C\|f\|_{L^{2}\left(Q_{T}\right)}\|v\|_{L^{2}\left(0, T ; H^{1}(\Omega)\right)} .
$$

For the second term, we have

$$
\begin{aligned}
&-\int_{0}^{T} a_{\mathrm{DG}}\left(\bar{u}_{h, \Delta t}, P_{h} v\right) d t=-\int_{0}^{T}\left(\left(\nabla_{h} \bar{u}_{h, \Delta t}, \nabla_{h} P_{h} v\right)+\epsilon\left(\left\{\nabla_{h} P_{h} v \cdot \mathbf{n}_{e}\right\},\left[\bar{u}_{h, \Delta t}\right]\right)_{\Gamma_{h} \cup \Gamma_{D}}\right) d t \\
&+\int_{0}^{T}\left(\left(\left\{\nabla_{h} \bar{u}_{h, \Delta t} \cdot \mathbf{n}_{e}\right\},\left[P_{h} v\right]\right)_{\Gamma_{h} \cup \Gamma_{D}}-\left(\frac{\sigma_{e}}{h_{e}}\left[\bar{u}_{h, \Delta t}\right],\left[P_{h} v\right]\right)_{\Gamma_{h} \cup \Gamma_{D}}\right) d t .
\end{aligned}
$$

We bound each term in the right-hand side. First, using (4.3) and (4.7), we obtain

$$
\int_{0}^{T}\left(\nabla_{h} \bar{u}_{h, \Delta t}, \nabla_{h} P_{h} v\right) d t \leq\left\|\nabla_{h} \bar{u}_{h, \Delta t}\right\|_{L^{2}\left(Q_{T}\right)}\left\|\nabla_{h} P_{h}(v)\right\|_{L^{2}\left(Q_{T}\right)} \leq C\|v\|_{L^{2}\left(0, T ; H^{1}(\Omega)\right)} .
$$


Next, by using equivalence of norms, we obtain

$$
\begin{aligned}
\int_{0}^{T}\left(\left\{\nabla_{h} P_{h} v \cdot \mathbf{n}_{e}\right\},\left[\bar{u}_{h, \Delta t}\right]\right)_{\Gamma_{h} \cup \Gamma_{D}} d t \leq C & \left(\int_{0}^{T} \sum_{e \in \Gamma_{h} \cup \Gamma_{D}} \frac{\sigma_{e}}{h_{e}}\left\|\left[\bar{u}_{h, \Delta t}\right]\right\|_{L^{2}(e)}^{2} d t\right)^{1 / 2} \\
& \times\left(\int_{0}^{T}\left\|\nabla_{h} P_{h} v\right\|_{L^{2}(\Omega)}^{2} d t\right)^{1 / 2} .
\end{aligned}
$$

Then (4.3) and (4.7) imply

$$
\int_{0}^{T}\left(\left\{\nabla_{h} P_{h} v \cdot \mathbf{n}_{e}\right\},\left[\bar{u}_{h, \Delta t}\right]\right)_{\Gamma_{h} \cup \Gamma_{D}} d t \leq C\|v\|_{L^{2}\left(0, T ; H^{1}(\Omega)\right)} .
$$

For the third term, using again an equivalence of norms, we write

$$
\begin{aligned}
& \int_{0}^{T}\left(\left\{\nabla_{h} \bar{u}_{h, \Delta t} \cdot \mathbf{n}_{e}\right\},\left[P_{h} v\right]\right)_{\Gamma_{h} \cup \Gamma_{D}} d t \leq C\left(\int_{0}^{T}\left\|\nabla_{h} \bar{u}_{h, \Delta t}\right\|_{L^{2}(\Omega)}^{2} d t\right)^{1 / 2}\left\|P_{h} v\right\|_{L^{2}\left(0, T ; X_{h}\right)} \\
& \leq C\|v\|_{L^{2}\left(0, T ; H^{1}(\Omega)\right)},
\end{aligned}
$$

by another application of (4.3) and (4.7). For the penalty term, we simply have

$$
\begin{aligned}
\int_{0}^{T}\left(\frac{\sigma_{e}}{h_{e}}\left[\bar{u}_{h, \Delta t}\right],\left[P_{h} v\right]\right)_{\Gamma_{h} \cup \Gamma_{D}} d t & \leq\left(\int_{0}^{T} \frac{\sigma_{e}}{h_{e}}\left\|\left[\bar{u}_{h, \Delta t}\right]\right\|_{L^{2}(e)}^{2} d t\right)^{1 / 2}\left\|P_{h} v\right\|_{L^{2}\left(0, T ; X_{h}\right)} \\
& \leq C\|v\|_{L^{2}\left(0, T ; H^{1}(\Omega)\right)},
\end{aligned}
$$

using again (4.3) and (4.7). Combining the bounds above we have obtained

$$
\int_{0}^{T}\left(\partial_{t} u_{h, \Delta t}, P_{h} v\right) d t \leq C\|v\|_{L^{2}\left(0, T ; H^{1}(\Omega)\right)},
$$

whence (4.8). $\square$

Lemma 4.2. The function $\partial_{t} u_{h, \Delta t}$ is uniformly bounded in $L^{2}\left(0, T ; X^{\prime}\right)$. More precisely, there is a constant $C$ independent of $h$ and $\Delta t$ such that

$$
\left\|\partial_{t} u_{h, \Delta t}\right\|_{L^{2}\left(0, T ; X^{\prime}\right)} \leq C .
$$

Proof. Pick an arbitrary $v \in L^{2}(0, T ; X)$ and write:

$$
\int_{0}^{T}\left(\partial_{t} u_{h, \Delta t}, v\right) d t=\int_{0}^{T}\left(\partial_{t} u_{h, \Delta t}, v-P_{h} v\right) d t+\int_{0}^{T}\left(\partial_{t} u_{h, \Delta t}, P_{h} v\right) d t .
$$

Since $P_{h} v$ is the $L^{2}$ projection of $v$ onto $X_{h}$ for almost every $t$, we have

$$
\int_{0}^{T}\left(\partial_{t} u_{h, \Delta t}, v-P_{h} v\right) d t=\sum_{n=0}^{N-1} \int_{t_{n}}^{t_{n+1}} \frac{1}{\Delta t}\left(u_{h}^{n+1}-u_{h}^{n}, v-P_{h} v\right) d t=0 .
$$


Thus from Lemma 4.1 we have, for all $v \in L^{2}(0, T ; X)$

$$
\int_{0}^{T}\left(\partial_{t} u_{h, \Delta t}, v\right) d t=\int_{0}^{T}\left(\partial_{t} u_{h, \Delta t}, P_{h} v\right) d t \leq C\|v\|_{L^{2}\left(0, T ; H^{1}(\Omega)\right)}
$$

which yields the result.

5. Convergence of $u_{h, \Delta t}$ and $\bar{u}_{h, \Delta t}$. The a priori bounds obtained in the previous section yield the following results.

TheOREm 5.1. There is a function $u \in L^{2}\left(Q_{T}\right)$ with $\partial_{t} u \in L^{2}\left(0, T ; X^{\prime}\right)$ such that

$$
u_{h, \Delta t} \text { and } \bar{u}_{h, \Delta t} \text { converge weakly to } u \text { in } L^{2}\left(Q_{T}\right) \text {, }
$$

and

$$
\partial_{t} u_{h, \Delta t} \text { converges weakly to } \partial_{t} u \text { in } L^{2}\left(0, T ; X^{\prime}\right) \text {. }
$$

Proof. From (4.6), there is a subsequence $\left(u_{h, \Delta t}\right)_{h, \Delta t}$ that converges weakly to a function $u$ in $L^{2}\left(Q_{T}\right)$. Bound (4.4) implies that there is a function $\bar{u}$ in $L^{2}\left(Q_{T}\right)$ such that, up to a subsequence

$$
\bar{u}_{h, \Delta t} \text { converges weakly to } \bar{u} \text { in } L^{2}\left(Q_{T}\right) \text {. }
$$

The difference

$$
u_{h, \Delta t}(t, x)-\bar{u}_{h, \Delta t}(t, x)=\left(u_{h}^{n+1}(x)-u_{h}^{n}(x)\right) \frac{t-t_{n+1}}{\Delta t}, \quad \forall(t, x) \in \Omega \times\left[t_{n}, t_{n+1}[,\right.
$$

gives

$$
\left\|u_{h, \Delta t}-\bar{u}_{h, \Delta t}\right\|_{\left.L^{2}\left(Q_{T}\right)\right)}^{2}==\frac{\Delta t}{3} \sum_{n=0}^{N-1}\left\|u_{h}^{n+1}-u_{h}^{n}\right\|_{L^{2}(\Omega)}^{2} .
$$

Using (3.3), we obtain

$$
\left\|u_{h, \Delta t}-\bar{u}_{h, \Delta t}\right\|_{L^{2}\left(Q_{T}\right)}^{2} \leq C \Delta t
$$

Hence

$$
\lim _{h, \Delta t \rightarrow 0}\left\|u_{h, \Delta t}-\bar{u}_{h, \Delta t}\right\|_{L^{2}\left(Q_{T}\right)}=0 .
$$

This yields that $u=\bar{u}$, and, up to a subsequence,

$$
\bar{u}_{h, \Delta t} \text { converges weakly to } u \text { in } L^{2}\left(Q_{T}\right) \text {. }
$$

Bound (4.9) implies that there is a function $w$ in $L^{2}\left(0, T ; X^{\prime}\right)$ and a subsequence, still denoted by $\left(u_{h, \Delta t}\right)_{h, \Delta t}$ such that

$$
\partial_{t} u_{h, \Delta t} \text { converges weakly to } w \text { in } L^{2}\left(0, T ; X^{\prime}\right) .
$$

A classical argument proves that, for all $v$ in $\mathcal{C}_{0}^{\infty}(0, T ; X)$, we have

$$
\langle w, v\rangle_{Q_{T}}=-\int_{0}^{T}\left(u, \partial_{t} v\right) d t=-\left(u, \partial_{t} v\right)_{Q_{T}}=\left\langle\partial_{t} u, v\right\rangle_{Q_{T}} .
$$


This means that, in the distributional sense,

$$
w=\partial_{t} u,
$$

which concludes the proof. $\mathrm{Q}$

Following [4], we introduce a discrete gradient as follows. For any face $e$ in $\Gamma_{h} \cup \Gamma_{D}$ and any function $\varphi \in L^{2}(e)$, we define the lifting $\boldsymbol{r}_{e}(\varphi) \in X_{h}^{d}$ by

$$
\int_{\Omega} \boldsymbol{r}_{e}(\varphi) \cdot \boldsymbol{v}_{h}=\int_{e}\left\{\boldsymbol{v}_{h} \cdot \mathbf{n}_{e}\right\} \varphi, \quad \forall \boldsymbol{v}_{h} \in X_{h}^{d}
$$

Then, for any $w_{h}$ in $X_{h}$, we set

$$
\boldsymbol{R}_{h}\left(\left[w_{h}\right]\right)=\sum_{e \in \Gamma_{h} \cup \Gamma_{D}} \boldsymbol{r}_{e}\left(\left[w_{h}\right]\right),
$$

and define the discrete symmetric gradient $\mathbf{G}_{h}\left(w_{h}\right)$ in each element $E$ by

$$
\left.\mathbf{G}_{h}\left(w_{h}\right)\right|_{E}=\left.\nabla w_{h}\right|_{E}-\left.\boldsymbol{R}_{h}\left(\left[w_{h}\right]\right)\right|_{E} .
$$

In particular, we have

$$
\left(\mathbf{G}_{h}\left(w_{h}\right), \mathbf{a}\right)=\left(\nabla_{h} w_{h}, \mathbf{a}\right)_{\mathcal{E}_{h}}-\left(\left[w_{h}\right],\left\{\mathbf{a} \cdot \mathbf{n}_{e}\right\}\right)_{\Gamma_{h} \cup \Gamma_{D}}, \quad \forall \mathbf{a} \in \mathbb{P}_{r}\left(\mathcal{E}_{h}\right)^{d} .
$$

Let $\bar{w}_{h, \Delta t}$ be the function, piecewise constant in time, defined by

$$
\left.\left.\bar{w}_{h, \Delta t}(t, x)=w_{h}^{n+1}(x), \quad \forall(t, x) \in \Omega \times\right] t_{n}, t_{n+1}\right] .
$$

We thus can extend the definition of the discrete gradient $\mathbf{G}_{h}\left(\bar{w}_{h, \Delta t}\right)$ :

$$
\left.\forall t \in] t_{n}, t_{n+1}\right], \mathbf{G}_{h}\left(\bar{w}_{h, \Delta t}\right)(t)=\mathbf{G}_{h}\left(w_{h}^{n+1}\right) .
$$

The discrete gradient satisfies the following important property.

Lemma 5.2. Denote by $Y$ the space of functions in $H^{1}(\Omega)$ whose trace vanishes on $\Gamma_{N}$, namely $Y=H_{0, \Gamma_{N}}^{1}(\Omega)$. Let $\mathbf{v}$ be a function in $L^{2}\left(0, T ; Y^{d}\right)$ and let $\mathbf{v}_{h}$ be any function in $L^{2}\left(0, T ; X_{h}^{d}\right) \cap L^{2}\left(0, T ; Y^{d}\right)$. Let $\left(w_{h}^{n}\right)_{n}$ be a sequence of functions in $X_{h}$. There exists a constant $C$ independent of $h$ and $\Delta t$ such that

$$
\begin{aligned}
\mid \int_{0}^{T}\left(\mathbf{G}_{h}\left(\bar{w}_{h, \Delta t}\right), \mathbf{v}\right)+\left(\bar{w}_{h, \Delta t}, \nabla\right. & \cdot \mathbf{v}) d t \mid \leq C\left\|\bar{w}_{h, \Delta t}\right\|_{L^{2}\left(0, T ; X_{h}\right)} \\
& \times\left(\left\|\mathbf{v}-\mathbf{v}_{h}\right\|_{L^{2}\left(Q_{T}\right)}^{2}+\sum_{e \in \Gamma_{h} \cup \Gamma_{D}} h_{e}\left\|\mathbf{v}-\mathbf{v}_{h}\right\|_{\left.L^{2}(e \times] 0, T\right)}^{2}\right)^{1 / 2} .
\end{aligned}
$$


Proof. By applying Green's formula, and the definitions (5.4) and (5.2), we obtain

$$
\int_{0}^{T}\left(\mathbf{G}_{h}\left(\bar{w}_{h, \Delta t}\right), \mathbf{v}\right) d t=\sum_{n=0}^{N-1} \int_{t_{n}}^{t_{n+1}}\left(-\left(w_{h}^{n+1}, \nabla \cdot \mathbf{v}\right)+\left(\left[w_{h}^{n+1}\right], \mathbf{v} \cdot \mathbf{n}_{e}\right)_{\Gamma_{h} \cup \Gamma_{D}}-\left(\mathbf{R}_{h}\left(\left[w_{h}^{n+1}\right]\right), \mathbf{v}\right)\right) d t .
$$

By using the fact that $\mathbf{v}_{h}$ belongs to $X_{h}^{d} \cap Y^{d}$ for each $t$, we write equivalently,

$$
\begin{aligned}
\int_{0}^{T}\left(\mathbf{G}_{h}\left(\bar{w}_{h, \Delta t}\right), \mathbf{v}\right) & d t=-\sum_{n=0}^{N-1} \int_{t_{n}}^{t_{n+1}}\left(w_{h}^{n+1}, \nabla \cdot \mathbf{v}\right) d t \\
+ & \sum_{n=0}^{N-1} \int_{t_{n}}^{t_{n+1}}\left(\left(\left[w_{h}^{n+1}\right],\left(\mathbf{v}-\mathbf{v}_{h}\right) \cdot \mathbf{n}_{e}\right)_{\Gamma_{h} \cup \Gamma_{D}}-\left(\mathbf{R}_{h}\left(\left[w_{h}^{n+1}\right]\right), \mathbf{v}-\mathbf{v}_{h}\right)\right) d t
\end{aligned}
$$

It is easy to show that

$$
\sum_{n=0}^{N-1} \Delta t\left\|\mathbf{R}_{h}\left(\left[w_{h}^{n+1}\right]\right)\right\|_{L^{2}(\Omega)}^{2} \leq C \int_{0}^{T} \sum_{e \in \Gamma_{h} \cup \Gamma_{D}} \frac{1}{h_{e}}\left\|\left[\bar{w}_{h, \Delta t}\right]\right\|_{L^{2}(e)}^{2} d t
$$

The result is then obtained.

The following lemma is a generalization of a result obtained in [4].

LEMMA 5.3. Let $\left(\bar{w}_{h, \Delta t}\right)_{h, \Delta t}$ be a sequence of functions, piecewise constant in time, satisfying

$$
\left\|\bar{w}_{h, \Delta t}\right\|_{L^{2}\left(0, T ; X_{h}\right)} \leq C,
$$

where $C$ is independent of $h$ and $\Delta t$. Assume that $\left(\bar{w}_{h, \Delta t}\right)_{h, \Delta t}$ converges weakly to a function $w$ in $L^{2}\left(Q_{T}\right)$. Then, the limit $w$ belongs to $L^{2}\left(0, T ; H^{1}(\Omega)\right)$ and the sequence $\left(\mathbf{G}_{h}\left(\bar{w}_{h, \Delta t}\right)\right)_{h, \Delta t}$ converges weakly to $\nabla w$ in $L^{2}\left(Q_{T}\right)^{d}$.

Proof. Using Lemma 5.2, we have for any function $\mathbf{v}$ in $L^{2}\left(0, T ; H_{0}^{1}(\Omega)^{d}\right)$

$\lim _{h, \Delta t \rightarrow 0} \int_{0}^{T}\left(\mathbf{G}_{h}\left(\bar{w}_{h, \Delta t}\right), \mathbf{v}\right) d t=-\lim _{h, \Delta t \rightarrow 0} \int_{0}^{T}\left(\bar{w}_{h, \Delta t}, \nabla \cdot \mathbf{v}\right) d t=-\int_{0}^{T}(w, \nabla \cdot \mathbf{v}) d t=\int_{0}^{T}\langle\nabla w, \mathbf{v}\rangle d t$.

In addition, we have, using (5.5)

$$
\left\|\mathbf{G}_{h}\left(\bar{w}_{h, \Delta t}\right)\right\|_{L^{2}\left(Q_{T}\right)} \leq C\left\|\bar{w}_{h, \Delta t}\right\|_{L^{2}\left(0, T ; X_{h}\right)} \leq C .
$$

Therefore the sequence $\left(\mathbf{G}_{h}\left(\bar{w}_{h, \Delta t}\right)\right)_{h, \Delta t}$ converges weakly to a function $\mathbf{z}$ in $L^{2}\left(Q_{T}\right)^{d}$ and (5.6) shows that the distributional derivative $\nabla w$ is in fact equal to $\mathbf{z}$.

The next theorem shows that the limit $u$ in Theorem 5.1 belongs to $L^{2}(0, T ; X)$.

THEOREM 5.4. With the notation of Theorem 5.1, we have

$$
u \in L^{2}(0, T ; X)
$$


Proof. First let us prove that $u$ takes its values in $H^{1}(\Omega)$. Let $\phi$ be an arbitrary function in $L^{2}\left(0, T ; \mathcal{C}_{0}^{\infty}(\Omega)^{d}\right)$. We have, from the weak convergence of $\bar{u}_{h, \Delta t}$ to $u$ in $L^{2}\left(Q_{T}\right)$ :

$$
-\int_{0}^{T}(u, \nabla \cdot \boldsymbol{\phi}) d t=\lim _{h, \Delta t \rightarrow 0}-\int_{0}^{T}\left(\bar{u}_{h, \Delta t}, \nabla \cdot \boldsymbol{\phi}\right) d t .
$$

Green's formula in each element $E$ implies

$$
-\int_{0}^{T}\left(\bar{u}_{h, \Delta t}, \nabla \cdot \boldsymbol{\phi}\right) d t=\int_{0}^{T}\left(\left(\nabla_{h} \bar{u}_{h, \Delta t}, \boldsymbol{\phi}\right)-\left(\boldsymbol{\phi} \cdot \mathbf{n}_{e},\left[\bar{u}_{h, \Delta t}\right]\right)_{\Gamma_{h}}\right) d t .
$$

Therefore

$$
\begin{aligned}
\left|-\int_{0}^{T}\left(\bar{u}_{h, \Delta t}, \nabla \cdot \boldsymbol{\phi}\right) d t\right| \leq & \int_{0}^{T}\left\|\nabla_{h} \bar{u}_{h, \Delta t}\right\|_{L^{2}(\Omega)}\|\phi\|_{L^{2}(\Omega)} d t \\
& +\int_{0}^{T}\left(\sum_{e \in \Gamma_{h}} \frac{\sigma_{e}}{h_{e}}\left\|\left[\bar{u}_{h, \Delta t}\right]\right\|_{L^{2}(e)}^{2}\right)^{1 / 2}\left(\sum_{e \in \Gamma_{h}} h_{e}\|\phi\|_{L^{2}(e)}^{2}\right)^{1 / 2} d t .
\end{aligned}
$$

By a standard trace theorem

$$
\sum_{e \in \Gamma_{h}} h_{e}\|\phi\|_{L^{2}(e)}^{2} \leq C\left(\|\phi\|_{L^{2}(\Omega)}^{2}+h^{2}\|\nabla \phi\|_{L^{2}(\Omega)}^{2}\right)
$$

Therefore the bound (4.3) yields

$$
\left|-\int_{0}^{T}\left(\bar{u}_{h, \Delta t}, \nabla \cdot \phi\right) d t\right| \leq C\left(\|\phi\|_{L^{2}\left(Q_{T}\right)}^{2}+h^{2}\|\nabla \phi\|_{L^{2}\left(Q_{T}\right)}^{2}\right)^{1 / 2}
$$

and passing to the limit as $h$ and $\Delta t$ tend to zero, we derive

$$
\left|-\int_{0}^{T}(u, \nabla \cdot \phi) d t\right| \leq C\|\phi\|_{L^{2}\left(Q_{T}\right)}
$$

This means that the distributional gradient of $u$ is in fact in $L^{2}\left(Q_{T}\right)^{d}$.

Next, In order to prove that the trace of $u$ vanishes on $\Gamma_{D}$, let $\phi$ and $\mathbf{v}$ be any functions respectively in $L^{2}(0, T)$ and $Y^{d}$, so that $\phi \mathbf{v}$ belongs to $L^{2}\left(0, T ; Y^{d}\right)$. We apply Lemma 5.2 to the sequence of functions $\left(u_{h}^{n}\right)_{n}$ and, considering that both $\mathbf{G}_{h}\left(\bar{u}_{h, \Delta t}\right)$ and $\bar{u}_{h, \Delta t}$ converge weakly, we obtain:

$$
\lim _{h, \Delta t \rightarrow 0} \int_{0}^{T}\left(\mathbf{G}_{h}\left(\bar{u}_{h, \Delta t}\right), \mathbf{v}\right) \phi d t=-\lim _{h, \Delta t \rightarrow 0} \int_{0}^{T}\left(\bar{u}_{h, \Delta t}, \nabla \cdot \mathbf{v}\right) \phi d t .
$$

Using Lemma 5.3, and passing to the limit, we have

$$
\int_{0}^{T}(\nabla u, \mathbf{v}) \phi d t=-\int_{0}^{T}(u, \nabla \cdot \mathbf{v}) \phi d t
$$


This implies for all $\phi \in L^{2}(0, T)$ and $\mathbf{v} \in Y$

$$
\int_{0}^{T}\left(u, \mathbf{v} \cdot \mathbf{n}_{e}\right)_{\Gamma_{D}} \phi d t=0,
$$

which in turn implies that the trace of $u$ vanishes on $\Gamma_{D}$.

With Theorem 5.1, we then recover the initial condition, namely

$$
u_{0}(\cdot)=u(0, \cdot)
$$

6. $L^{p}$ convergence results for broken Sobolev spaces. In this section, we obtain convergence in $L^{p}$ of a uniformly bounded sequence of discrete functions in broken Sobolev spaces. The result is valid independently of the choice of the boundary conditions.

THEOREM 6.1. Let $p_{0}$ be the critical exponent of the Sobolev embedding defined by (3.7) and let $p<p_{0}$. Let $\left(v_{h}\right)_{h}$ be a sequence of functions in $X_{h}$, uniformly bounded:

$$
\left\|v_{h}\right\|_{X_{h}} \leq C
$$

Then, if $\left|\Gamma_{D}\right|=0$, there is a function $v \in H^{1}(\Omega)$ such that $\left(v_{h}-\int_{\Omega} v_{h}\right)_{h}$ converges strongly to $v$ in the $L^{p}$ norm. In addition, if $\left|\Gamma_{D}\right|>0$, the function $v$ belongs to $H_{0, \Gamma_{D}}^{1}(\Omega)$ and $\left(v_{h}\right)_{h}$ converges strongly to $v$ in the $L^{p}$ norm.

The proof of this result contains intermediate steps that can be useful per se. The discontinuous function $v_{h}$ is transformed into an $H^{1}$ function by means of three operators. The first operator $L_{h}$ reduces the degree of the polynomials to one; it is simply a standard Lagrange interpolant of degree one applied locally to $v_{h}$ in each element with no global continuity requirement. The next operator $C_{h}$ regularizes $L_{h}\left(v_{h}\right)$ at the center of each face to transform $L_{h}\left(v_{h}\right)$ into a Crouzeix-Raviart element (see [3]). Finally, $C_{h}\left(v_{h}\right)$ is globally regularized by a Scott-Zhang type element (see [10]), denoted $R_{h}\left(v_{h}\right)$. Details of the constructions of $C_{h}$ and $R_{h}$ are given below.

To construct $C_{h}$, consider first an interior face $e$ shared by $E_{1}^{e}$ and $E_{2}^{e}$ (normal $\mathbf{n}_{e}$ points from $E_{1}^{e}$ to $E_{2}^{e}$ ), let $\mathbf{b}_{e}$ denote the midpoint of $e$ in $2 \mathrm{D}$ and the barycenter of $e$ in $3 \mathrm{D}$. Then $\lambda_{e}$ is a discontinuous piecewise polynomial of degree one, uniquely defined by

$$
\left.\lambda_{e}\left(\mathbf{b}_{e}\right)\right|_{E_{1}^{e}}=1,\left.\quad \lambda_{e}\left(\mathbf{b}_{e}\right)\right|_{E_{2}^{e}}=0, \quad \lambda_{e}\left(\mathbf{b}_{\gamma}\right)=0, \quad \forall \gamma \in \Gamma_{h} \cup \Gamma_{D}, \quad \gamma \neq e .
$$

The process is similar for a boundary face $e$ adjacent to $E^{e}$; we define $\lambda_{e}$, a discontinuous piecewise polynomial of degree one, by

$$
\left.\lambda_{e}\left(\mathbf{b}_{e}\right)\right|_{E^{e}}=1, \quad \lambda_{e}\left(\mathbf{b}_{\gamma}\right)=0, \quad \forall \gamma \in \Gamma_{h} \cup \Gamma_{D}, \quad \gamma \neq e .
$$

Then $C_{h}\left(v_{h}\right)$ is defined by

$$
C_{h}\left(v_{h}\right)=L_{h}\left(v_{h}\right)-\sum_{e \in \Gamma_{h} \cup \Gamma_{D}}\left(\frac{1}{|e|} \int_{e}\left[L_{h}\left(v_{h}\right)\right]\right) \lambda_{e} .
$$


By construction, $C_{h}\left(v_{h}\right)$ is continuous at the center of each interior $e$ and vanishes at the center of each $e$ in $\Gamma_{D}$.

We now regularize $C_{h}\left(v_{h}\right)$ by constructing a function that is a polynomial of degree one in each element, belongs globally to $H^{1}(\Omega)$, and is of the Scott-Zhang type [10]. Let $\mathcal{P}_{h}$ denote the set of vertices of $\mathcal{E}_{h}$, including those on the boundary $\partial \Omega$. For each vertex $\mathbf{a} \in \mathcal{P}_{h}$, let $\phi_{\mathbf{a}}$ be the nodal basis function of degree one associated with $\mathbf{a}$, and let $\mathbf{b}_{a}$ be the center of a face with vertex $\mathbf{a}$, chosen so that the face is not contained in $\Gamma_{N}$. In addition, if $\left|\Gamma_{D}\right|>0$ and if a lies on $\Gamma_{D}$, the face is chosen so that it belongs to $\Gamma_{D}$. Then $R_{h}\left(v_{h}\right)$ is defined by

$$
R_{h}\left(v_{h}\right)=\sum_{\mathbf{a} \in \mathcal{P}_{h}} C_{h}\left(v_{h}\right)\left(\mathbf{b}_{a}\right) \phi_{\mathbf{a}}
$$

By construction, $R_{h}\left(v_{h}\right)$ belongs to $X$.

The following lemma collects useful approximation properties of these operators. Its proof is easy and given in the appendix.

LEMma 6.2. Let the mesh be regular in the sense of Ciarlet [2]. Then, there exists a constant $C$ independent of $h$, such that

$$
\begin{gathered}
\left\|v_{h}-L_{h}\left(v_{h}\right)\right\|_{X_{h}} \leq C\left\|\nabla_{h} v_{h}\right\|_{L^{2}(\Omega)}, \quad \forall v_{h} \in X_{h}, \\
\left\|v_{h}-C_{h}\left(v_{h}\right)\right\|_{X_{h}} \leq C\left\|v_{h}\right\|_{X_{h}}, \quad \forall v_{h} \in X_{h}, \\
\left\|\nabla R_{h}\left(v_{h}\right)\right\|_{L^{2}(\Omega)} \leq C\left\|v_{h}\right\|_{X_{h}}, \quad \forall v_{h} \in X_{h} .
\end{gathered}
$$

For each $p \leq p_{0}$, the critical exponent of Sobolev's embedding, there exists a constant $C$ independent of $h$, such that

$$
\begin{gathered}
\left\|v_{h}-L_{h}\left(v_{h}\right)\right\|_{L^{p}(\Omega)} \leq C h^{1-d\left(\frac{1}{2}-\frac{1}{p}\right)}\left\|\nabla_{h} v_{h}\right\|_{L^{2}(\Omega)}, \quad \forall v_{h} \in X_{h}, \\
\left\|v_{h}-C_{h}\left(v_{h}\right)\right\|_{L^{p}(\Omega)} \leq C h^{1-d\left(\frac{1}{2}-\frac{1}{p}\right)}\left\|v_{h}\right\|_{X_{h}}, \quad \forall v_{h} \in X_{h}, \\
\left\|v_{h}-R_{h}\left(v_{h}\right)\right\|_{L^{p}(\Omega)} \leq C h^{1-d\left(\frac{1}{2}-\frac{1}{p}\right)}\left\|v_{h}\right\|_{X_{h}}, \quad \forall v_{h} \in X_{h} .
\end{gathered}
$$

We can now prove Theorem 6.1.

Proof. All weak convergences below are up to subsequences.

We consider two cases according to the measure of $\Gamma_{D}$. If $\left|\Gamma_{D}\right|>0$, it follows from (3.10) with $p=2$, that $v_{h}$ is uniformly bounded in $L^{2}(\Omega)$ and hence there is $v \in L^{2}(\Omega)$ such that $v_{h}$ converges weakly to $v$ in $L^{2}(\Omega)$. Similarly, there is a function $w \in H_{0, \Gamma_{D}}^{1}(\Omega)$ such that $R_{h}\left(v_{h}\right)$ converges weakly to $w$ in $H^{1}(\Omega)$. This means that $R_{h}\left(v_{h}\right)$ converges strongly to $w$ in $L^{p}(\Omega)$ for all $p<p_{0}$. But (6.7) implies that $v_{h}-R_{h}\left(v_{h}\right)$ converges strongly to zero in $L^{p}(\Omega)$ and therefore on one hand $v=w$ and on the other hand, $v_{h}$ converges strongly to $v$ in $L^{p}(\Omega)$.

Next, consider the case $\left|\Gamma_{D}\right|=0$; then $\|\cdot\|_{X_{h}}$ is a semi-norm, and $v_{h}$ is defined up to an additive constant. Let

$$
\tilde{v}_{h}=v_{h}-\frac{1}{|\Omega|} \int_{\Omega} R_{h}\left(v_{h}\right)
$$


It is easy to see that by construction

$$
R_{h}\left(\tilde{v}_{h}\right)=R_{h}\left(v_{h}\right)-\frac{1}{|\Omega|} \int_{\Omega} R_{h}\left(v_{h}\right) .
$$

Thus $R_{h}\left(\tilde{v}_{h}\right)$ belongs to $H^{1}(\Omega)$ and $\int_{\Omega} R_{h}\left(\tilde{v}_{h}\right)=0$, and hence by Peetre-Tartar's lemma (see [8], [11] and Theorem 2.1 in [6])

$$
\left\|R_{h}\left(\tilde{v}_{h}\right)\right\|_{L^{p}(\Omega)} \leq C\left\|\nabla R_{h}\left(\tilde{v}_{h}\right)\right\|_{L^{2}(\Omega)}=C\left\|\nabla R_{h}\left(v_{h}\right)\right\|_{L^{2}(\Omega)} \leq C\left\|v_{h}\right\|_{X_{h}} \leq C .
$$

Therefore there is $w \in H^{1}(\Omega)$ such that $\left(R_{h}\left(\tilde{v}_{h}\right)\right)_{h}$ converges weakly to $w$ in $H^{1}(\Omega)$ and strongly to $w$ in $L^{p}(\Omega)$ for $p<p_{0}$. Reverting to $\tilde{v}_{h}$, we write

$$
\left\|\tilde{v}_{h}\right\|_{L^{p}(\Omega)} \leq\left\|\tilde{v}_{h}-R_{h}\left(\tilde{v}_{h}\right)\right\|_{L^{p}(\Omega)}+\left\|R_{h}\left(\tilde{v}_{h}\right)\right\|_{L^{p}(\Omega)} .
$$

But

$$
\tilde{v}_{h}-R_{h}\left(\tilde{v}_{h}\right)=v_{h}-R_{h}\left(v_{h}\right)
$$

and therefore, again by (6.7),

$$
\left\|\tilde{v}_{h}\right\|_{L^{p}(\Omega)} \leq\left\|v_{h}-R_{h}\left(v_{h}\right)\right\|_{L^{p}(\Omega)}+C\left\|v_{h}\right\|_{X_{h}} \leq C\left\|v_{h}\right\|_{X_{h}} .
$$

Hence there is a function $v \in L^{p}(\Omega)$ such that $\tilde{v}_{h}$ converges weakly to $v$ in $L^{p}(\Omega)$. In addition, it stems from

$$
\left\|\tilde{v}_{h}-w\right\|_{L^{p}(\Omega)} \leq\left\|\tilde{v}_{h}-R_{h}\left(\tilde{v}_{h}\right)\right\|_{L^{p}(\Omega)}+\left\|R_{h}\left(\tilde{v}_{h}\right)-w\right\|_{L^{p}(\Omega)},
$$

that $\left(\tilde{v}_{h}\right)_{h}$ converges strongly to $w$ in $L^{p}(\Omega)$. This implies that $v=w$. Summing up, the sequence

$$
\left(v_{h}-\int_{\Omega} R_{h}\left(v_{h}\right)\right)_{h}
$$

converges strongly to $v$ in $L^{p}(\Omega)$, and $v \in H^{1}(\Omega)$. Since by (6.7),

$$
\frac{1}{|\Omega|}\left\|\int_{\Omega}\left(R_{h}\left(v_{h}\right)-v_{h}\right)\right\|_{L^{p}(\Omega)} \leq|\Omega|^{1 / p-1 / 2}\left\|R_{h}\left(v_{h}\right)-v_{h}\right\|_{L^{2}(\Omega)} \leq C|\Omega|^{1 / p-1 / 2} h\left\|v_{h}\right\|_{X_{h}},
$$

the conclusion holds for $\left(v_{h}-\frac{1}{|\Omega|} \int_{\Omega} v_{h}\right)_{h}$.

7. Proof of Theorem 3.1. For two sequences of functions in $X_{h}, \tilde{v}_{h}=\left(v_{h}^{n}\right)_{n}$ and $\tilde{w}_{h}=\left(w_{h}^{n}\right)_{n}$, define the bilinear form associated with the left-hand side of (3.1):

$$
A_{h}\left(\tilde{w}_{h}, \tilde{v}_{h}\right)=\sum_{n=0}^{N-1}\left(w_{h}^{n+1}-w_{h}^{n}, v_{h}^{n+1}\right)+\Delta t \sum_{n=0}^{N-1} a_{\mathrm{DG}}\left(w_{h}^{n+1}, v_{h}^{n+1}\right) .
$$

Lemma 7.1. Let $\tilde{v}_{h}=\left(v_{h}^{n}\right)_{n}$ be a sequence of functions in $X_{h}$. There is a constant $C$ independent of $h, \Delta t$ and $\tilde{v}_{h}$ such that

$$
A_{h}\left(\tilde{v}_{h}, \tilde{v}_{h}\right) \geq C \sum_{n=1}^{N} \Delta t\left\|v_{h}^{n}\right\|_{X_{h}}^{2}+\frac{1}{2}\left\|v_{h}^{N}\right\|_{L^{2}(\Omega)}^{2}-\frac{1}{2}\left\|v_{h}^{0}\right\|_{L^{2}(\Omega)}^{2} .
$$


Proof. The proof is well-known. We give it for completeness. By definition

$$
A_{h}\left(\tilde{v}_{h}, \tilde{v}_{h}\right)=\sum_{n=0}^{N-1}\left(v_{h}^{n+1}-v_{h}^{n}, v_{h}^{n+1}\right)+\Delta t \sum_{n=0}^{N-1} a_{\mathrm{DG}}\left(v_{h}^{n+1}, v_{h}^{n+1}\right) .
$$

On one hand, the coercivity of $a_{\mathrm{DG}}$ implies

$$
a_{\mathrm{DG}}\left(v_{h}^{n+1}, v_{h}^{n+1}\right) \geq C\left\|\tilde{v}_{h}\right\|_{X_{h}}^{2} .
$$

On the other hand, the first term satisfies

$$
\sum_{n=0}^{N-1}\left(v_{h}^{n+1}-v_{h}^{n}, v_{h}^{n+1}\right) \geq \frac{1}{2} \sum_{n=0}^{N-1}\left(\left\|v_{h}^{n+1}\right\|_{L^{2}(\Omega)}^{2}-\left\|v_{h}^{n}\right\|_{L^{2}(\Omega)}^{2}\right)=\frac{1}{2}\left\|v_{h}^{N}\right\|_{L^{2}(\Omega)}^{2}-\frac{1}{2}\left\|v_{h}^{0}\right\|_{L^{2}(\Omega)}^{2} .
$$

This concludes the proof.

Ideally, the desired strong convergence should be deduced by substituting into (7.1) the difference between $u_{h}$ and some interpolant of the limit function $u$. But, considering the low regularity of $u$, we replace it by a smooth function and argue by density. More precisely, we know that $u$ belongs to $W(0, T)$, see $(2.3)$. Since the smooth functions are dense in $W(0, T)$, we can find a sequence $\left(\phi_{k}\right)_{k}$ in $\mathcal{C}^{\infty}([0, T] ; X)$ such that

$$
\phi_{k} \text { converges strongly to } u \text { in } W(0, T) \text {, }
$$

and the embedding (2.4) implies that $\phi_{k}$ converges strongly to $u$ in $\mathcal{C}^{0}\left([0, T] ; L^{2}(\Omega)\right)$. In other words, for each $\delta>0$, there exists $k_{0}$ such that for all $k \geq k_{0}$,

$$
\left\|\phi_{k}-u\right\|_{W(0, T)} \leq \delta
$$

and

$$
\sup _{t \in[0, T]}\left\|\phi_{k}(t)-u(t)\right\|_{L^{2}(\Omega)} \leq \delta .
$$

Let $I_{h, \Delta t} \phi_{k}$ be an interpolant of $\phi_{k}$, of the Scott-Zhang type [10], continuous in time and space, vanishing on $\Gamma_{D}$ and satisfying

$I_{h, \Delta t} \phi_{k}$ converges strongly to $\phi_{k}$ in $L^{2}(0, T ; X)$,

$\partial_{t}\left(I_{h, \Delta t} \phi_{k}\right)$ converges strongly to $\partial_{t} \phi_{k}$ in $L^{2}\left(Q_{T}\right)$.

This implies

$$
I_{h, \Delta t} \phi_{k} \text { converges strongly to } \phi_{k} \text { in } \mathcal{C}^{0}\left([0, T] ; L^{2}(\Omega)\right) \text {. }
$$

We set

$$
\bar{I}_{h, \Delta t} \phi_{k}(x)=I_{h, \Delta t} \phi_{k}\left(t_{n+1}, x\right), \quad \forall(t, x) \in \Omega \times\left[t_{n}, t_{n+1}\right] .
$$


It can be easily checked that, since $I_{h, \Delta t} \phi_{k}$ belongs to a finite dimensional space in time, $\bar{I}_{h, \Delta t} \phi_{k}$ also converges strongly to $\phi_{k}$ in $L^{2}(0, T ; X)$, and moreover

$$
\left\|\bar{I}_{h, \Delta t} \phi_{k}-\phi_{k}\right\|_{L^{2}\left(0, T ; H^{1}(\Omega)\right)} \leq C\left\|I_{h, \Delta t} \phi_{k}-\phi_{k}\right\|_{L^{2}\left(0, T ; H^{1}(\Omega)\right)}
$$

Denote by $\tilde{I}_{h, \Delta t} \phi_{k}$ the sequence of functions $\left(I_{h, \Delta t} \phi_{k}\left(t_{n}, \cdot\right)\right)_{n}$ and by $\tilde{u}_{h}$ the sequence of functions $\left(u_{h}^{n}(\cdot)\right)_{n}$. We apply Lemma 7.1 to the sequence $\tilde{u}_{h}-\tilde{I}_{h, \Delta t} \phi_{k_{0}}$ with the index $k_{0}$ chosen in (7.2). We have, since the interpolant does not jump

$$
\begin{array}{r}
C\left(\sum_{n=1}^{N} \Delta t\left(\left\|\nabla_{h}\left(u_{h}^{n}-I_{h, \Delta t} \phi_{k_{0}}\left(t_{n}, \cdot\right)\right)\right\|_{L^{2}(\Omega)}^{2}+\sum_{e \in \Gamma_{h} \cup \Gamma_{D}} \frac{\sigma_{e}}{h_{e}}\left\|\left[u_{h}^{n}\right]\right\|_{L^{2}(e)}^{2}\right)\right)+\frac{1}{2}\left\|u_{h}^{N}-I_{h, \Delta t} \phi_{k_{0}}(T, \cdot)\right\|_{L^{2}(\Omega)}^{2} \\
\leq A_{h}\left(\tilde{u}_{h}-\tilde{I}_{h, \Delta t} \phi_{k_{0}}, \tilde{u}_{h}-\tilde{I}_{h, \Delta t} \phi_{k_{0}}\right)+\frac{1}{2}\left\|u_{h}^{0}-I_{h, \Delta t} \phi_{k_{0}}(0, \cdot)\right\|_{L^{2}(\Omega)}^{2} .
\end{array}
$$

This implies

$$
\begin{array}{r}
C\left\|\bar{u}_{h, \Delta t}-\bar{I}_{h, \Delta t} \phi_{k_{0}}\right\|_{L^{2}\left(0, T ; X_{h}\right)}^{2}+\frac{1}{2}\left\|u_{h}^{N}-I_{h, \Delta t} \phi_{k_{0}}(T, \cdot)\right\|_{L^{2}(\Omega)}^{2} \leq \\
A_{h}\left(\tilde{u}_{h}-\tilde{I}_{h, \Delta t} \phi_{k_{0}}, \tilde{u}_{h}-\tilde{I}_{h, \Delta t} \phi_{k_{0}}\right) \\
+\frac{1}{2}\left\|u_{h}^{0}-I_{h, \Delta t} \phi_{k_{0}}(0, \cdot)\right\|_{L^{2}(\Omega)}^{2} .
\end{array}
$$

The right-hand side of (7.6) can be split into three terms. The first one is evaluated by the expression (3.1) of the scheme:

$$
A_{h}\left(\tilde{u}_{h}, \tilde{u}_{h}-\tilde{I}_{h, \Delta t} \phi_{k_{0}}\right)=\Delta t \sum_{n=0}^{N-1}\left(f^{n+1}, u_{h}^{n+1}-I_{h, \Delta t} \phi_{k_{0}}\left(t_{n+1}, \cdot\right)\right)=\int_{0}^{T}\left(\bar{f}_{\Delta t}, \bar{u}_{h, \Delta t}-\bar{I}_{h, \Delta t} \phi_{k_{0}}\right) d t
$$

The weak convergence of $\bar{u}_{h, \Delta t}$ in $L^{2}\left(Q_{T}\right)$, the strong convergence of $\bar{I}_{h, \Delta t} \phi_{k_{0}}$ to $\phi_{k_{0}}$ in $\mathcal{C}^{0}\left([0, T] ; L^{2}(\Omega)\right)$ and the strong convergence of $\bar{f}_{\Delta t}$ to $f$ imply that

$$
\lim _{h, \Delta t \rightarrow 0} A_{h}\left(\tilde{u}_{h}, \tilde{u}_{h}-\tilde{I}_{h, \Delta t} \phi_{k_{0}}\right)=\int_{0}^{T}\left(f, u-\phi_{k_{0}}\right) d t
$$

The next lemma evaluates the limit of the second term.

LEMMA 7.2. We have

$$
\lim _{h, \Delta t \rightarrow 0} A_{h}\left(\tilde{I}_{h, \Delta t} \phi_{k_{0}}, \tilde{u}_{h}-\tilde{I}_{h, \Delta t} \phi_{k_{0}}\right)=\int_{0}^{T}\left(\partial_{t} \phi_{k_{0}}, u-\phi_{k_{0}}\right) d t+\int_{0}^{T}\left(\nabla \phi_{k_{0}}, \nabla\left(u-\phi_{k_{0}}\right)\right) d t
$$


Proof. Since $I_{h, \Delta t} \phi_{k_{0}}\left(t_{n}, \cdot\right)$ belongs to $X$, the expression of $A_{h}$ simplifies:

$$
\begin{aligned}
A_{h}\left(\tilde{I}_{h, \Delta t} \phi_{k_{0}}, \tilde{u}_{h}-\tilde{I}_{h, \Delta t} \phi_{k_{0}}\right)= & \sum_{n=0}^{N-1}\left(\left(I_{h, \Delta t} \phi_{k_{0}}\left(t_{n+1}\right)-I_{h, \Delta t} \phi_{k_{0}}\left(t_{n}\right), u_{h}^{n+1}-I_{h, \Delta t} \phi_{k_{0}}\left(t_{n+1}\right)\right)\right. \\
& +\Delta t\left(\nabla I_{h, \Delta t} \phi_{k_{0}}\left(t_{n+1}\right), \nabla_{h} u_{h}^{n+1}-\nabla I_{h, \Delta t} \phi_{k_{0}}\left(t_{n+1}\right)\right) \\
& \left.-\Delta t\left(\left\{\nabla I_{h, \Delta t} \phi_{k_{0}}\left(t_{n+1}\right) \cdot \mathbf{n}_{e}\right\},\left[u_{h}^{n+1}-I_{h, \Delta t} \phi_{k_{0}}\left(t_{n+1}\right)\right]\right)_{\Gamma_{h} \cup \Gamma_{D}}\right) .
\end{aligned}
$$

Therefore the first term in the above sum can be rewritten as

$$
\begin{aligned}
& \sum_{n=0}^{N-1}\left(I_{h, \Delta t} \phi_{k_{0}}\left(t_{n+1}\right)-I_{h, \Delta t} \phi_{k_{0}}\left(t_{n}\right), u_{h}^{n+1}-I_{h, \Delta t} \phi_{k_{0}}\left(t_{n+1}\right)\right) \\
&=\int_{0}^{T}\left(\partial_{t} I_{h, \Delta t} \phi_{k_{0}}, \bar{u}_{h, \Delta t}-\bar{I}_{h, \Delta t} \phi_{k_{0}}\right) d t
\end{aligned}
$$

By assumption, $\partial_{t} I_{h, \Delta t} \phi_{k_{0}}$ converges strongly to $\partial_{t} \phi_{k_{0}}$ in $L^{2}\left(Q_{T}\right)$. We also have shown the weak convergence of $\bar{u}_{h, \Delta t}$ to $u$ in $L^{2}\left(Q_{T}\right)$. From $(7.4), \bar{I}_{h, \Delta t} \phi_{k_{0}}$ converges strongly to $\phi_{k_{0}}$ in $L^{2}\left(Q_{T}\right)$. Therefore, the first term in the right-hand side of (7.9) converges to

$$
\int_{0}^{T}\left(\partial_{t} \phi_{k_{0}}, u-\phi_{k_{0}}\right) d t=\int_{0}^{T}\left\langle\partial_{t} \phi_{k_{0}}, u-\phi_{k_{0}}\right\rangle d t
$$

We first rewrite the sum of the two remaining terms as

$$
\int_{0}^{T}\left(\left(\nabla \bar{I}_{h, \Delta t} \phi_{k_{0}}, \nabla_{h} \bar{u}_{h, \Delta t}-\nabla \bar{I}_{h, \Delta t} \phi_{k_{0}}\right)-\left(\left\{\nabla \bar{I}_{h, \Delta t} \phi_{k_{0}} \cdot \mathbf{n}_{e}\right\},\left[\bar{u}_{h, \Delta t}-\bar{I}_{h, \Delta t} \phi_{k_{0}}\right]\right)_{\Gamma_{h} \cup \Gamma_{D}}\right) .
$$

Using the discrete gradient $\mathbf{G}_{h}\left(\bar{u}_{h, \Delta t}-\bar{I}_{h, \Delta t} \phi_{k_{0}}\right)$, this expression becomes

$$
\begin{aligned}
\sum_{n=1}^{N} \Delta t\left(\left(\nabla I_{h, \Delta t} \phi_{k_{0}}\left(t_{n}\right), \nabla_{h} u_{h}^{n}-\nabla I_{h, \Delta t} \phi_{k_{0}}\left(t_{n}\right)\right)\right. & \left.-\left(\left\{\nabla I_{h, \Delta t} \phi_{k_{0}}\left(t_{n}\right) \cdot \mathbf{n}_{e}\right\},\left[u_{h}^{n}-I_{h, \Delta t} \phi_{k_{0}}\left(t_{n}\right)\right]\right)_{\Gamma_{h} \cup \Gamma_{D}}\right) \\
= & \int_{0}^{T}\left(\mathbf{G}_{h}\left(\bar{u}_{h, \Delta t}-\bar{I}_{h, \Delta t} \phi_{k_{0}}\right), \nabla \bar{I}_{h, \Delta t} \phi_{k_{0}}\right) d t .
\end{aligned}
$$

Next we observe that the sequence $\left(\bar{u}_{h, \Delta t}-\bar{I}_{h, \Delta t} \phi_{k_{0}}\right)_{h, \Delta t}$ is uniformly bounded in $L^{2}\left(0, T ; X_{h}\right)$. It stems from Lemma 5.3 that the sequence $\left(\mathbf{G}_{h}\left(\bar{u}_{h, \Delta t}-\bar{I}_{h, \Delta t} \phi_{k_{0}}\right)\right)_{h, \Delta t}$ converges weakly to $\nabla u-\nabla \phi_{k_{0}}$ in $L^{2}\left(Q_{T}\right)$. From (7.4), we see that the sequence $\left(\bar{I}_{h, \Delta t} \phi_{k_{0}}\right)_{h, \Delta t}$ converges strongly to $\phi_{k_{0}}$ in $L^{2}\left(0, T ; H^{1}(\Omega)\right)$. We then conclude that

$$
\lim _{h, \Delta t \rightarrow 0} \int_{0}^{T}\left(\mathbf{G}_{h}\left(\bar{u}_{h, \Delta t}-\bar{I}_{h, \Delta t} \phi_{k_{0}}\right), \nabla \bar{I}_{h, \Delta t} \phi_{k_{0}}\right) d t=\int_{0}^{T}\left(\nabla \phi_{k_{0}}, \nabla\left(u-\phi_{k_{0}}\right)\right) d t,
$$

which combined with (7.10) gives (7.8). 
For the last term in the right-hand side of (7.6) that involves the solution at the initial time, we first see that, since $u_{h}^{0}$ is the $L^{2}$ projection of $u_{0}$

$$
\lim _{h \rightarrow 0}\left\|u_{h}^{0}-u_{0}\right\|_{L^{2}(\Omega)}=0 .
$$

Then the strong convergence of $I_{h, \Delta t} \phi_{k_{0}}(0, \cdot)$ to $\phi_{k_{0}}(0, \cdot)$ in $L^{2}(\Omega)$ implies

$$
\lim _{h \rightarrow 0}\left\|u_{h}^{0}-I_{h, \Delta t} \phi_{k_{0}}(0, \cdot)\right\|_{L^{2}(\Omega)}=\left\|u_{0}-\phi_{k_{0}}(0, \cdot)\right\|_{L^{2}(\Omega)}
$$

By collecting the above results, and combining with (5.7), (7.7) and (7.8), we obtain on one hand that for each $\varepsilon>0$ there exist $h_{\varepsilon}$ and $\Delta t_{\varepsilon}$ such that for all $h \leq h_{\varepsilon}$ and $\Delta t \leq \Delta t_{\varepsilon}$,

$$
\begin{aligned}
& \mid A_{h}\left(\tilde{u}_{h}-\tilde{I}_{h, \Delta t} \phi_{k_{0}}, \tilde{u}_{h}-\tilde{I}_{h, \Delta t} \phi_{k_{0}}\right)+\frac{1}{2}\left\|u_{h}^{0}-I_{h, \Delta t} \phi_{k_{0}}(0, \cdot)\right\|_{L^{2}(\Omega)}^{2} \\
& -\int_{0}^{T}\left(\left(f, u-\phi_{k_{0}}\right)+\left\langle\partial_{t} \phi_{k_{0}}, u-\phi_{k_{0}}\right\rangle+\left(\nabla \phi_{k_{0}}, \nabla\left(u-\phi_{k_{0}}\right)\right)\right) d t-\frac{1}{2}\left\|u(0, \cdot)-\phi_{k_{0}}(0, \cdot)\right\|_{L^{2}(\Omega)}^{2} \mid \\
& \leq \varepsilon .
\end{aligned}
$$

On the other hand, by applying (7.2) and (7.3),

$$
\begin{aligned}
& \int_{0}^{T}\left(\left(f, u-\phi_{k_{0}}\right)+\left\langle\partial_{t} \phi_{k_{0}}, u-\phi_{k_{0}}\right\rangle+\left(\nabla \phi_{k_{0}}, \nabla\left(u-\phi_{k_{0}}\right)\right)\right) d t+\frac{1}{2}\left\|u(0, \cdot)-\phi_{k_{0}}(0, \cdot)\right\|_{L^{2}(\Omega)}^{2} \\
& \leq\|f\|_{L^{2}\left(Q_{T}\right)}\left\|u-\phi_{k_{0}}\right\|_{L^{2}\left(Q_{T}\right)}+\left\|\partial_{t} \phi_{k_{0}}\right\|_{L^{2}\left(0, T ; X^{\prime}\right)}\left\|u-\phi_{k_{0}}\right\|_{L^{2}\left(0, T ; H^{1}(\Omega)\right)} \\
& +\left\|\phi_{k_{0}}\right\|_{L^{2}\left(0, T ; H^{1}(\Omega)\right)}\left\|u-\phi_{k_{0}}\right\|_{L^{2}\left(0, T ; H^{1}(\Omega)\right)}+\frac{1}{2}\left\|u(0, \cdot)-\phi_{k_{0}}(0, \cdot)\right\|_{L^{2}(\Omega)}^{2} \\
& \leq \delta\left(\|f\|_{L^{2}\left(Q_{T}\right)}+\left\|\partial_{t} \phi_{k_{0}}\right\|_{L^{2}\left(0, T ; X^{\prime}\right)}+\left\|\phi_{k_{0}}\right\|_{L^{2}\left(0, T ; H^{1}(\Omega)\right)}\right)+\frac{1}{2} \delta^{2} \leq C_{1} \delta
\end{aligned}
$$

for some constant $C_{1}$ independent of $\delta$. In particular, by choosing $\varepsilon=\delta$, we infer from (7.11) and (7.12) that for all $h \leq h_{\delta}$ and $\Delta t \leq \Delta t_{\delta}$

$$
\left|A_{h}\left(\tilde{u}_{h}-\tilde{I}_{h, \Delta t} \phi_{k_{0}}, \tilde{u}_{h}-\tilde{I}_{h, \Delta t} \phi_{k_{0}}\right)+\frac{1}{2}\left\|u_{h}^{0}-I_{h, \Delta t} \phi_{k_{0}}(0, \cdot)\right\|_{L^{2}(\Omega)}^{2}\right| \leq\left(1+C_{1}\right) \delta
$$

with the constant $C_{1}$ of (7.12). By reverting to (7.6), (7.13) implies that, for all $h \leq h_{\delta}$ and $\Delta t \leq \Delta t_{\delta}$

$$
\left\|\bar{u}_{h, \Delta t}-\bar{I}_{h, \Delta t} \phi_{k_{0}}\right\|_{L^{2}\left(0, T ; X_{h}\right)}^{2}+\left\|u_{h}^{N}-I_{h, \Delta t} \phi_{k_{0}}(T, \cdot)\right\|_{L^{2}(\Omega)}^{2} \leq C_{2} \delta
$$

for another constant $C_{2}$ depending only on $C_{1}$ and the constant of (7.6). From the triangle inequality, (7.14), the convergence of $I_{h, \Delta t}$, and (7.2) we deduce that, by possibly restricting further $h_{\delta}$ and $\Delta t_{\delta}$, there holds for all $h \leq h_{\delta}$ and $\Delta t \leq \Delta t_{\delta}$

$$
\begin{aligned}
\left\|\bar{u}_{h, \Delta t}-u\right\|_{L^{2}\left(0, T ; X_{h}\right)} \leq & \left\|\bar{u}_{h, \Delta t}-\bar{I}_{h, \Delta t} \phi_{k_{0}}\right\|_{L^{2}\left(0, T ; X_{h}\right)}+\left\|\bar{I}_{h, \Delta t} \phi_{k_{0}}-\phi_{k_{0}}\right\|_{L^{2}\left(0, T ; X_{h}\right)}+\left\|\phi_{k_{0}}-u\right\|_{L^{2}\left(0, T ; X_{h}\right)} \\
& \leq C_{3} \delta
\end{aligned}
$$


with another constant $C_{3}$ independent of $\delta$. This is precisely the convergence (3.4). Of course, (7.14) implies the same convergence for $u_{h}^{N}-u(T)$ in $L^{2}(\Omega)$, and the same argument can be applied to the value at any intermediate point, thus implying (3.5).

Finally, by reverting to (7.5), the convergence (3.4) implies that the jumps tend to zero:

$$
\lim _{h, \Delta t \rightarrow 0} \Delta t \sum_{n=1}^{N} \sum_{e \in \Gamma_{h} \cup \Gamma_{D}} \frac{\sigma_{e}}{h_{e}}\left\|\left[u_{h}^{n}\right]\right\|_{L^{2}(e)}^{2}=0 .
$$

8. Passing to the limit. Let $\phi$ be a smooth function in space and in time satisfying $\phi(T)=0$, interpolated with the same interpolant as above. From (4.2), we have by choosing $v_{h}=\bar{I}_{h, \Delta t} \phi$ (recall it is the piecewise constant in time function):

$$
\int_{0}^{T}\left(\partial_{t} u_{h, \Delta t}, \bar{I}_{h, \Delta t} \phi\right)+\int_{0}^{T} a_{\mathrm{DG}}\left(\bar{u}_{h, \Delta t}, \bar{I}_{h, \Delta t} \phi\right)=\int_{0}^{T}\left(\bar{f}_{\Delta t}, \bar{I}_{h, \Delta t} \phi\right) .
$$

Clearly the right-hand side converges to

$$
\lim _{h, \Delta t \rightarrow 0} \int_{0}^{T}\left(\bar{f}_{\Delta t}, \bar{I}_{h, \Delta t} \phi\right)=\int_{0}^{T}(f, \phi) .
$$

Since $\partial_{t} u_{h, \Delta t}$ converges weakly to $\partial_{t} u$ in $L^{2}\left(0, T ; X^{\prime}\right)$ and $\bar{I}_{h, \Delta t} \phi$ converges strongly to $\phi$ in $L^{2}(0, T ; X)$ we have

$$
\lim _{h, \Delta t \rightarrow 0} \int_{0}^{T}\left(\partial_{t} u_{h, \Delta t}, \bar{I}_{h, \Delta t} \phi\right)=\int_{0}^{T}\left\langle\partial_{t} u, \phi\right\rangle .
$$

We expand $a_{\mathrm{DG}}$, considering that $\bar{I}_{h, \Delta t} \phi$ does not have any jumps in space:

$$
\int_{0}^{T} a_{\mathrm{DG}}\left(\bar{u}_{h, \Delta t}, \bar{I}_{h, \Delta t} \phi\right)=\int_{0}^{T}\left(\nabla_{h} \bar{u}_{h, \Delta t}, \nabla \bar{I}_{h, \Delta t} \phi\right)+\epsilon\left(\left\{\nabla \bar{I}_{h, \Delta t} \phi\right\} \cdot \mathbf{n}_{e},\left[\bar{u}_{h, \Delta t}\right]\right)_{\Gamma_{h} \cup \Gamma_{D}} .
$$

Since $\nabla_{h} \bar{u}_{h, \Delta t}$ converges strongly to $\nabla u$ in $L^{2}\left(Q_{T}\right)$, and $\nabla \bar{I}_{h, \Delta t} \phi$ converges strongly to $\nabla \phi$ in $L^{2}\left(Q_{T}\right)$, we have

$$
\lim _{h, \Delta t \rightarrow 0} \int_{0}^{T}\left(\nabla_{h} \bar{u}_{h, \Delta t}, \nabla \bar{I}_{h, \Delta t} \phi\right)=\int_{0}^{T}(\nabla u, \nabla \phi) .
$$

It follows from (7.15) that

$$
\lim _{h, \Delta t \rightarrow 0} \int_{0}^{T} \sum_{e \in \Gamma_{h} \cup \Gamma_{D}} \frac{\sigma_{e}}{h_{e}}\left\|\left[\bar{u}_{h, \Delta t}\right]\right\|_{L^{2}(e)}^{2}=0 .
$$

We write by equivalence of norms

$$
\int_{0}^{T}\left(\left\{\nabla \bar{I}_{h, \Delta t} \phi\right\} \cdot \mathbf{n}_{e},\left[\bar{u}_{h, \Delta t}\right]\right)_{\Gamma_{h} \cup \Gamma_{D}} \leq C\left(\int_{0}^{T} \sum_{e \in \Gamma_{h} \cup \Gamma_{D}} \frac{\sigma_{e}}{h_{e}}\left\|\left[\bar{u}_{h, \Delta t}\right]\right\|_{L^{2}(e)}^{2}\right)^{1 / 2}\left\|\nabla \bar{I}_{h, \Delta t} \phi\right\|_{L^{2}\left(Q_{T}\right)} .
$$


Therefore,

$$
\lim _{h, \Delta t \rightarrow 0} \int_{0}^{T} \epsilon\left(\left\{\nabla \bar{I}_{h, \Delta t} \phi\right\} \cdot \mathbf{n}_{e},\left[\bar{u}_{h, \Delta t}\right]\right)_{\Gamma_{h} \cup \Gamma_{D}}=0 .
$$

Hence by collecting these limits, the function $u$ satisfies (2.1).

REMARK 8.1. In the case of quadrilateral or hexahedral elements, since DG functions require no continuity, $\mathbb{P}_{r}(E)$ can also be used, instead of $\mathcal{F}^{-1}\left(\mathbb{Q}_{r}(\hat{E})\right)$. In this case, the interpolants cannot be continuous in space; thus their jumps do not vanish, and must be estimated. But those estimates are straightforward, they increase somewhat the length of the proofs, but do not change the results.

\section{Appendix.}

9.1. Proof of Theorem 6.2. The estimates (6.2) and (6.5) are easy consequences of the approximation properties of the standard Lagrange interpolant $L_{h}$ and the smoothness of $v_{h}$ in the interior of each element.

Next, we turn to $C_{h}$. To derive the interior estimate (6.3), we consider $\left\|C_{h}\left(u_{h}\right)-L_{h}\left(u_{h}\right)\right\|_{X_{h}}$. We have for an element $E$ :

$$
\left\|\nabla\left(C_{h}\left(u_{h}\right)-L_{h}\left(u_{h}\right)\right)\right\|_{L^{2}(E)} \leq \sum_{e \in \partial E \cap\left(\Gamma_{h} \cup \Gamma_{D}\right)} \frac{1}{|e|}\left(\int_{e}\left[L_{h}\left(u_{h}\right)\right]\right)\left\|\nabla \lambda_{e}\right\|_{L^{2}(E)} .
$$

Therefore, we infer from (6.2) that

$$
\begin{gathered}
\left\|\nabla_{h}\left(C_{h}\left(u_{h}\right)-L_{h}\left(u_{h}\right)\right)\right\|_{L^{2}(\Omega)} \leq C\left(\sum_{e \in \Gamma_{h} \cup \Gamma_{D}} \frac{\sigma_{e}}{h_{e}}\left\|\left[L_{h}\left(u_{h}\right)\right]\right\|_{L^{2}(e)}^{2}\right)^{1 / 2} \\
\leq C\left(\left(\sum_{e \in \Gamma_{h} \cup \Gamma_{D}} \frac{\sigma_{e}}{h_{e}}\left\|\left[L_{h}\left(u_{h}\right)-u_{h}\right]\right\|_{L^{2}(e)}^{2}\right)^{1 / 2}+\left(\sum_{e \in \Gamma_{h} \cup \Gamma_{D}} \frac{\sigma_{e}}{h_{e}}\left\|\left[u_{h}\right]\right\|_{L^{2}(e)}^{2}\right)^{1 / 2}\right) \leq C\left\|u_{h}\right\|_{X_{h}},
\end{gathered}
$$

and

$$
\left\|\nabla_{h}\left(u_{h}-C_{h}\left(u_{h}\right)\right)\right\|_{L^{2}(\Omega)} \leq C\left\|u_{h}\right\|_{X_{h}} .
$$

To bound the jumps, let $e$ be an arbitrary interior face, shared by $E_{1}^{e}$ and $E_{2}^{e}$, the argument being similar for a boundary face $e \in \Gamma_{D}$. By definition,

$$
\left.\left[C_{h}\left(u_{h}\right)-L_{h}\left(u_{h}\right)\right]\right|_{e}=-\left.\sum_{\gamma \in \Gamma_{h} \cup \Gamma_{D}}\left(\frac{1}{|\gamma|} \int_{\gamma}\left[L_{h}\left(u_{h}\right)\right]\right)\left[\lambda_{\gamma}\right]\right|_{e} .
$$

Denote by $\mathcal{S}_{e}$ the set of faces in $\left(\partial E_{1}^{e} \cup \partial E_{2}^{e}\right) \cap\left(\Gamma_{h} \cup \Gamma_{D}\right)$ :

$$
\left\|\left[C_{h}\left(u_{h}\right)-L_{h}\left(u_{h}\right)\right]\right\|_{L^{2}(e)} \leq \sum_{\gamma \in \mathcal{S}_{e}}\left(\frac{1}{|\gamma|} \int_{\gamma}\left[L_{h}\left(u_{h}\right)\right]\right)\left\|\left[\lambda_{\gamma}\right]\right\|_{L^{2}(e)} .
$$


Thus

$$
\frac{\sigma_{e}}{h_{e}}\left\|\left[C_{h}\left(u_{h}\right)-I_{h}\left(u_{h}\right)\right]\right\|_{L^{2}(e)}^{2} \leq C \frac{\sigma_{e}}{h_{e}} \sum_{\gamma \in \mathcal{S}_{e}}\left\|\left[L_{h}\left(u_{h}\right)\right]\right\|_{L^{2}(\gamma)}^{2},
$$

and

$$
\sum_{e \in \Gamma_{h}} \frac{\sigma_{e}}{h_{e}}\left\|\left[C_{h}\left(u_{h}\right)-I_{h}\left(u_{h}\right)\right]\right\|_{L^{2}(e)}^{2} \leq C \sum_{e \in \Gamma_{h} \cup \Gamma_{D}} \frac{\sigma_{e}}{h_{e}}\left\|\left[L_{h}\left(u_{h}\right)\right]\right\|_{L^{2}(e)}^{2} .
$$

For the $L^{p}$ estimate (6.6), it suffices to show that

$$
\left\|C_{h}\left(v_{h}\right)-L_{h}\left(v_{h}\right)\right\|_{L^{p}(\Omega)} \leq C h^{1-d\left(\frac{1}{2}-\frac{1}{p}\right)}\left\|v_{h}\right\|_{X_{h}} .
$$

On an arbitrary mesh element $E$,

$$
\begin{gathered}
\left\|C_{h}\left(u_{h}\right)-L_{h}\left(u_{h}\right)\right\|_{L^{p}(E)} \leq \sum_{e \in \partial E \cap\left(\Gamma_{h} \cup \Gamma_{D}\right)} \frac{1}{|e|}\left|\int_{e}\left[L_{h}\left(u_{h}\right)\right]\right|\left\|\lambda_{e}\right\|_{L^{p}(E)} \\
\quad \leq C h^{1-d\left(\frac{1}{2}-\frac{1}{p}\right)}\left(\sum_{e \in \partial E \cap\left(\Gamma_{h} \cup \Gamma_{D}\right)} \frac{\sigma_{e}}{h_{e}}\left\|\left[L_{h}\left(u_{h}\right)\right]\right\|_{L^{2}(e)}^{2}\right)^{1 / 2} \cdot
\end{gathered}
$$

By applying Jensen's inequality, valid here since $p \geq 2$, we obtain

$$
\left\|C_{h}\left(u_{h}\right)-L_{h}\left(u_{h}\right)\right\|_{L^{p}(\Omega)} \leq C h^{1-d\left(\frac{1}{2}-\frac{1}{p}\right)}\left(\sum_{e \in \Gamma_{h} \cup \Gamma_{D}} \frac{\sigma_{e}}{h_{e}}\left\|\left[L_{h}\left(u_{h}\right)\right]\right\|_{L^{2}(e)}^{2}\right)^{1 / 2},
$$

whence (6.6).

Now, we turn to $R_{h}$. The stability and approximation properties of the Scott-Zhang operator imply that

$$
\left\|\nabla R_{h}\left(u_{h}\right)\right\|_{L^{2}(\Omega)} \leq C\left\|\nabla_{h} C_{h}\left(u_{h}\right)\right\|_{L^{2}(\Omega)}
$$

and on an arbitrary mesh element $E$

$$
\left\|C_{h}\left(u_{h}\right)-R_{h}\left(u_{h}\right)\right\|_{L^{p}(E)}^{p} \leq C h_{E}^{p+d-\frac{d p}{2}} \sum_{k=1}^{j}\left\|\nabla_{h} C_{h}\left(u_{h}\right)\right\|_{L^{2}\left(E_{j}\right)}^{p}
$$

where $\cup_{k=1}^{j} E_{k}$ is a macro-element containing $E$, where the values of the function are taken to evaluate $R_{h}$. Then (6.7) follows from the results obtained for $C_{h}\left(v_{h}\right)$.

9.2. Proof of Theorem 3.3. We write

$$
\left\|v_{h}\right\|_{L^{p}(\Omega)} \leq\left\|v_{h}-R_{h}\left(v_{h}\right)\right\|_{L^{p}(\Omega)}+\left\|R_{h}\left(v_{h}\right)\right\|_{L^{p}(\Omega)} .
$$

From (6.7), we obtain

$$
\left\|v_{h}\right\|_{L^{p}(\Omega)} \leq C h^{1-d\left(\frac{1}{2}-\frac{1}{p}\right)}\left\|v_{h}\right\|_{X_{h}}+\left\|R_{h}\left(v_{h}\right)\right\|_{L^{p}(\Omega)} .
$$


By Sobolev's embedding,

$$
\left\|R_{h}\left(v_{h}\right)\right\|_{L^{p}(\Omega)} \leq C\left\|R_{h}\left(v_{h}\right)\right\|_{H^{1}(\Omega)} .
$$

We know that

$$
\left\|\nabla R_{h}\left(v_{h}\right)\right\|_{L^{2}(\Omega)} \leq C\left\|v_{h}\right\|_{X_{h}}
$$

and using (6.7) with $p=2$

$$
\left\|R_{h}\left(v_{h}\right)\right\|_{L^{2}(\Omega)} \leq C\left\|v_{h}\right\|_{X_{h}}+\left\|v_{h}\right\|_{L^{2}(\Omega)} .
$$

Therefore,

$$
\left\|R_{h}\left(v_{h}\right)\right\|_{L^{p}(\Omega)} \leq C\left(\left\|v_{h}\right\|_{X_{h}}+\left\|v_{h}\right\|_{L^{2}(\Omega)}\right),
$$

and we obtain (3.9).

Next, assume $\left|\Gamma_{D}\right|>0$. This implies

$$
\left\|R_{h}\left(v_{h}\right)\right\|_{L^{2}(\Omega)} \leq C\left\|\nabla R_{h}\left(v_{h}\right)\right\|_{L^{2}(\Omega)} \leq C\left\|v_{h}\right\|_{X_{h}},
$$

which, in turn, implies

$$
\left\|R_{h}\left(v_{h}\right)\right\|_{L^{p}(\Omega)} \leq C\left\|v_{h}\right\|_{X_{h}} .
$$

This concludes the proof of (3.10).

Finally, we consider the case $\left|\Gamma_{D}\right|=0$. As in the proof of Theorem 6.1, we define

$$
\tilde{v}_{h}=v_{h}-\frac{1}{|\Omega|} \int_{\Omega} R_{h}\left(v_{h}\right)
$$

and we obtain, in an exact fashion, the bound

$$
\left\|\tilde{v}_{h}\right\|_{L^{p}(\Omega)} \leq\left\|v_{h}-R_{h}\left(v_{h}\right)\right\|_{L^{p}(\Omega)}+C\left\|v_{h}\right\|_{X_{h}}
$$

Using (6.7) we have

$$
\left\|\tilde{v}_{h}\right\|_{L^{p}(\Omega)} \leq C\left\|v_{h}\right\|_{X_{h}} .
$$

Next, we write

$$
\left\|v_{h}-\frac{1}{|\Omega|} \int_{\Omega} v_{h}\right\|_{L^{p}(\Omega)} \leq\left\|\tilde{v}_{h}\right\|_{L^{p}(\Omega)}+\left\|\frac{1}{|\Omega|} \int_{\Omega}\left(R_{h}\left(v_{h}\right)-v_{h}\right)\right\|_{L^{p}(\Omega)} .
$$

This implies

$$
\left\|v_{h}-\frac{1}{|\Omega|} \int_{\Omega} v_{h}\right\|_{L^{p}(\Omega)} \leq C\left\|v_{h}\right\|_{X_{h}}+|\Omega|^{1 / p-1 / 2}\left\|R_{h}\left(v_{h}\right)-v_{h}\right\|_{L^{2}(\Omega)} .
$$

Using (6.7) with $p=2$, we obtain (3.11).

\section{REFERENCES}


[1] S. Brenner, Poincaré-Friedrichs inequalities for piecewise $H^{1}$ functions, SIAM Journal on Numerical Analysis, 41 (2003), pp. 306-324.

[2] P. G. Ciarlet, The finite element method for elliptic problems, North-Holland, Amsterdam, 1978.

[3] M. Crouzeix and P.-A. Raviart, Conforming and nonconforming finite element methods for solving the stationary Stokes equations., RAIRO Numerical Analysis, 193 (1973), pp. 33-75.

[4] D. Di Pietro And A. ERn, Discrete functional analysis tools for discontinuous Galerkin methods with application to the incompressible Navier-Stokes equations, Mathematics of Computation, 79 (2010), pp. 1303-1330.

[5] V. Girault And P.-A. Raviart, Finite element approximation of the navier-stokes equations, Lecture Notes in Mathematics, Berlin Springer Verlag, 749 (1979).

[6] V. Girault and P.-A. Raviart, Finite element methods for Navier-Stokes equations: theory and algorithms, vol. 5, Springer-Verlag, 1986.

[7] V. Girault and B. Riviere, DG approximation of coupled Navier-Stokes and Darcy equations by Beaver-Joseph-Saffman interface condition, SIAM Journal on Numerical Analysis, 47 (2009), pp. 2052-2089.

[8] J. Peetre, Espaces d'interpolation et théorème de soboleff, in Annales de l'institut Fourier, vol. 16, Institut Fourier, 1966, pp. 279-317.

[9] B. RIviere, Discontinuous Galerkin Methods for Solving Elliptic and Parabolic Equations: Theory and Implementation, SIAM, 2008.

[10] L. R. Scott And S. Zhang, Finite element interpolation of nonsmooth functions satisfying boundary conditions, Mathematics of Computation, 54 (1990), pp. 483-493.

[11] L. TARTAR, Topics in nonlinear analysis, volume 13 of publications mathématiques dorsay 78, Université de Paris-Sud Département de Mathématique, Orsay, (1978).

[12] J. Wloka, Partial Differential Equations, Cambridge University, 1987. 